\title{
CENTRAL NERVOUS SYSTEM LYMPHOMA IN IMMUNOCOMPROMISED PATIENT
}

\author{
Muhammad Atif Naveed \\ Department of Radiology, Shaukat Khanum Memorial Cancer Hospital and Research Centre, Lahore, Pakistan \\ Received: 22 December 2017 / Accepted: 28 March 2018
}

A 3-year-old boy, with pre-B acute lymphoblastic leukaemia, on chemotherapy, was hospitalized for the antibiotic and antifungal therapy for the febrile neutropenia (white blood cell count $0.71 \times 10^{3} \mathrm{ul}$ ). During the course of treatment, computed tomography (CT) maxillofacial was performed for the evaluation of fungal sinusitis, and subsequently, magnetic resonance imaging (MRI) brain was advised for possible intracranial extension of disease.

On the MRI brain, there was an incidental note of a large mass located in the right periventricular/periatrial white matter which demonstrated diffusion restriction on driving while intoxicated (DWI) and analogue-to-digital converter (ADC) images, no contrast enhancement on T1 fat-saturated post-gadolinium images, mild T2/FLAIR hyperintensity and punctate foci of haemorrhages on gradient-recalled echo images [Figure 1]. This mass was demonstrating mass effect on the adjacent brain and ventricle. There was no leptomeningeal disease or peripheral vasogenic oedema. On the basis of imaging, we favoured the diagnosis of central nervous system lymphoma (CNSL) in this immunecompromised patient. This lesion was demonstrating mass effect, hypercellularity (as indicated by significant diffusion restriction), intratumoural haemorrhagic foci, periventricular location and no contrast enhancement. The patient died the same day of the MRI scan due to febrile neutropenia. The other diagnostic considerations were infection, infarct and metastasis. The images did not show peripheral hypointense rim on T2-weighted images, no ring-like enhancement or central diffusion restriction which is are key features of an intracerebral abscess. Furthermore, the lesion demonstrated mass effect and does not involve overlying cortex, rendering the diagnosis of infarct less likely. The differential diagnosis for metastasis was less likely, as there was no peripheral vasogenic oedema or contrast enhancement.

Correspondence: Dr. Muhammad Atif Naveed, Department of Radiology, Shaukat Khanum Memorial Cancer Hospital Research Centre, Lahore, Pakistan.

Email: atifnaveed@skm.org.pk

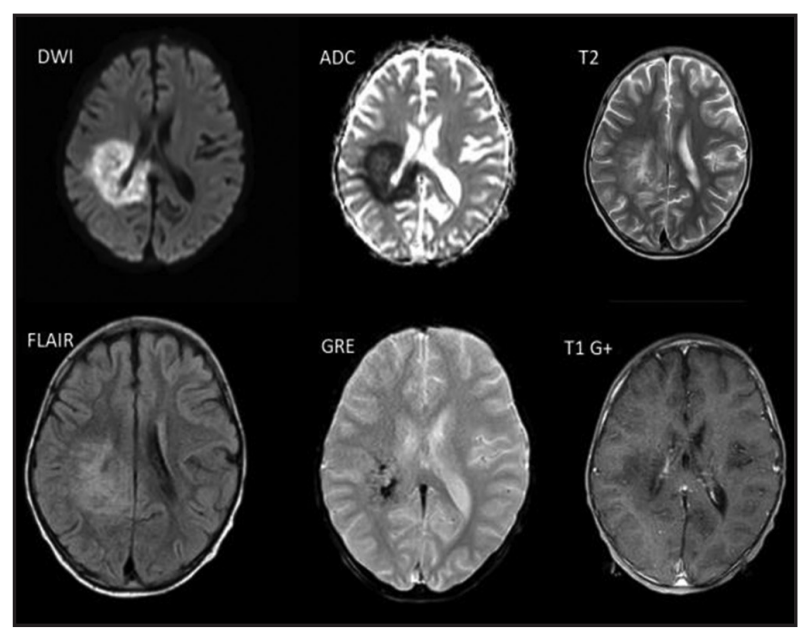

Figure 1: Axial driving while intoxicated and analogue-todigital converter magnetic resonance (MR) images show diffusion restriction in the right periatrial tumour; increased signal intensity relative to grey matter in the tumour with mild peritumoural oedema (T2 and FLAIR MR images) and mass effect on the ventricle. Axial gradient-recalled echo MR image reveals foci of haemorrhages within the tumour. Axial T1 post-gadolinium fat-saturated MR images show no contrast enhancement in this immunocompromised patient

Immunosuppression, congenital or acquired, increases the risk of developing CNS lymphoma. ${ }^{[1]}$ Primary CNSL in immunosuppressed patients presents with variable imaging features. The lesions can be non-enhancing ${ }^{[2]}$, as in our case; furthermore, irregular or ring enhancement patterns can be seen addition to haemorrhage and necrosis in these patients. ${ }^{[2]}$

DWI indicates the diffusion of water molecules in the tissues; diffusion within the tumour is known to be a surrogate marker of tumour cellularity because densely packed cells constitute a barrier to water diffusion. The CNS lymphomas are highly cellular tumours, causing restriction of the water diffusion, making them appear hyperintense on DWI and hypointense on ADC maps. Our case has the limitation that the patient did not survive long enough to have advanced MRI or positron-emission tomography imaging or, brain biopsy or cerebrospinal fluid studies to confirm the findings; however, the above-mentioned MR 
features and mass effect by the tumour are important to narrow the differential diagnosis. In addition to the MRI brainwith and without gadolinium, the complete physical, neurological and ophthalmological examination are necessary. ${ }^{[3]}$ The secondary CNS lymphoma and primary CNS lymphoma can be differentiated on the basis of bone marrow biopsy, testicular ultrasound and CT of the chest, abdomen and pelvis. Stereotactic biopsy of the tumour is the gold standard for the final diagnosis of lymphoma. ${ }^{[3]}$

\section{Conflict of Interest}

The authors declare that they have no conflict of interest.

\section{References}

1. Haldorsen IS, Espeland A, Larsson EM. Central nervous system lymphoma: Characteristic findings on traditional and advanced imaging. AJNR Am J Neuroradiol 2011;32:984-92.

2. Johnson BA, Fram EK, Johnson PC, et al. The variable MR appearance of primary lymphoma of the central nervous system: Comparison with histopathologic features. AJNR Am J Neuroradiol 1997;18:563-72.

3. Baraniskin A, Deckert M, Schulte-Altedorneburg G, et al. Current strategies in the diagnosis of diffuse large B-cell lymphoma of the central nervous system. Br J Haematol 2012;156:421-32. 